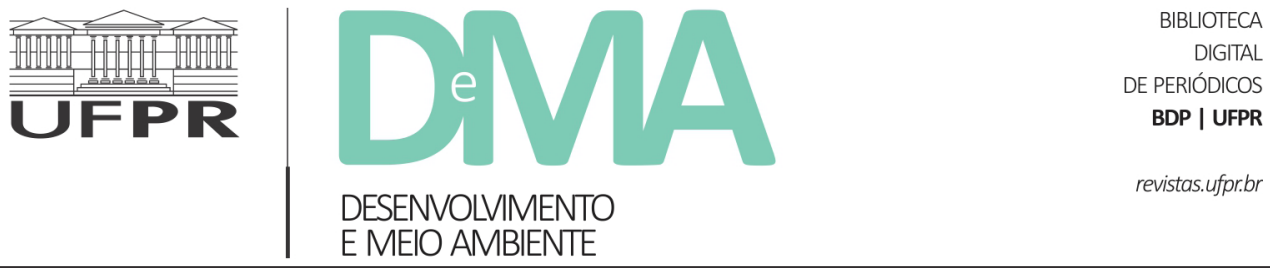

\title{
Gestão descentralizada de soluções de esgotamento sanitário no Brasil: aspectos conceituais, normativos e alternativas tecnológicas
}

\section{Decentralized management of sewage solutions in Brazil: conceptual, regulatory aspects and technological alternatives}

Tayane Cristiele Rodrigues MESQUITA ${ }^{1}$, André Pereira ROSA ${ }^{1 *}$, Uende Aparecida Figueiredo GOMES ${ }^{2}$, Alisson Carraro BORGES ${ }^{1}$

${ }^{1}$ Universidade Federal de Viçosa (UFV), Viçosa, MG, Brasil.

${ }^{2}$ Universidade Federal de Minas Gerais (UFMG), Belo Horizonte, MG, Brasil.

*E-mail de contato: andrerosa@ufv.br

Artigo recebido em 15 de abril de 2020, versão final aceita em 6 de outubro de 2020, publicado em 26 de março de 2021.

RESUMO: Diante da dificuldade de fornecer acesso adequado ao esgotamento sanitário em regiões não atendidas por sistemas centralizados, a adoção de sistemas descentralizados mostra-se relevante para a superação do déficit. Sendo assim, buscou-se nesse estudo analisar os aspectos conceituais relativos à utilização de sistemas descentralizados no país e discutir questões relacionadas à gestão desses sistemas à luz do marco legal e institucional da área de saneamento. Para tanto, inicialmente, definiram-se os contextos nos quais os sistemas descentralizados são mais utilizados, a partir de revisão da literatura. Posteriormente, foram avaliadas alternativas de tratamento utilizadas no saneamento descentralizado. Nesse ponto, foram analisados dados de estações de tratamento de esgotos (ETEs) em operação no país, disponibilizados pela Agência Nacional de Águas e Saneamento Básico (ANA). Por fim, foi realizada uma análise documental do marco legal e institucional que regulamenta o saneamento brasileiro e suas interfaces com a gestão descentralizada de esgotos. Verificou-se que, no Brasil, as tecnologias descentralizadas mais utilizadas são os reatores UASB e tanques sépticos seguidos por filtros anaeróbios (TS+FAN). No entanto, a utilização dessas tecnologias, bem como de outras soluções descentralizadas carece de regulamentação federal que defina a responsabilidade pela gestão dos sistemas. A nível estadual, apenas dois estados brasileiros possuem regulamentações que definem tal responsabilidade. Sobre os TS+FAN, três estados, localizados no Sudeste e Sul do país, possuem leis que normatizam a utilização dessas tecnologias na ausência de redes públicas de coleta. A discussão aponta que para superar a precariedade e ausência de acesso ao esgotamento sanitário é necessário empreender esforços na ampliação do atendimento dos serviços, sobretudo em áreas rurais, periurbanas e de difícil acesso ao sistema centralizado. Neste contexto, a combinação entre sistemas centralizados e descentralizados pode ser 
uma solução adequada para superação do déficit em esgotamento sanitário.

Palavras-chave: sistemas descentralizados; saneamento; tratamento de esgotos.

ABSTRACT: Adoption of decentralized systems is an alternative for sewage treatment even in the face of hardships in regions not covered by centralized systems. Nevertheless, the discussion on the approach of decentralized systems in terms of regulatory frameworks is still incipient. Therefore, this study aimed at investigating the decentralized systems application in Brazil, as well as discussing the management of these systems in the light of legislation. Firstly, the ideal conditions of decentralized systems were evaluated based on a comprehensive literature review. Afterwards a documentary analysis of sanitation legislation was carried out beyond its interfaces with decentralized management. The more indicated technological alternatives were evaluated by using data from sewage treatment plants (STPs) in Brazil according to the Brazilian National Water and SanitationAgency (ANA). At last, a document analysis of the legal landmark and its interfaces with decentralized systems management was carried out. In Brazil it was verified that the most useful decentralized systems were UASB reactors and septic tanks followed by anaerobic filters $(\mathrm{ST}+\mathrm{AF})$, although the responsible for the decentralized systems are scarcely defined. At the state level in Brazil only two states have guidelines that define responsibilities for the management of decentralized systems. In addition, only three states located in the regions Southeast and South have regulations for decentralized systems in locations where sewage collection networks are absent. It could be concluded that is required more efforts to expand sanitation services especially in rural and periurban areas, as well as in an area of difficult access. In this context the combination between centralized and decentralized systems could be a proper alternative to overcome the deficit in sanitation.

Keywords: decentralized systems; sanitation; sewage treatment.

\section{Introdução}

Em 2010, o acesso ao esgotamento sanitário foi considerado direito humano pela Organização da Nações Unidas (ONU). No entanto, este direito tem sido recorrentemente violado, uma vez que existem 673 milhões de pessoas no mundo ainda praticando a defecação a céu aberto (WHO, 2019). Na América Latina e Caribe, 15,5 milhões de pessoas não têm acesso a banheiros. Nesses locais, pessoas com renda mais baixa, em áreas rurais e indígenas, são as mais afetadas (WHO, 2019).

No Brasil, o Plano Nacional de Saneamento Básico (Plansab), aprovado em novembro de 2013, por meio Decreto $n^{\circ} 8.141$, caracterizou como atendi- mento apropriado ao esgotamento sanitário o acesso no qual há coleta de esgotos, seguida de tratamento, bem como o uso de tanque séptico. De acordo com o Plansab (2013), apenas 39,4\% da população brasileira têm acesso ao esgotamento sanitário, sendo que, desse total, $12 \%$ referem-se a sistemas declarados como tanques sépticos. Já o Programa Saneamento Brasil Rural (PSBR), aprovado em dezembro de 2019 por meio do Portaria $\mathrm{n}^{\mathrm{o}} 3.174$, ampliou o conceito de atendimento apropriado ao esgotamento sanitário, considerando também a utilização de fossa seca, nos casos de indisponibilidade hídrica (PSBR, 2019). No entanto, segundo o PSBR (2019), nas áreas rurais brasileiras ${ }^{1}$, o acesso apropriado ao esgotamento sanitário é de apenas $20,6 \%$.

\footnotetext{
${ }^{1}$ Importante observar que o PSBR apresenta uma definição de rural própria considerando critérios de densidade demográfica e vizinhança. A partir da metodologia aplicada, tem-se, como resultado, uma população residente em 11.192.096 domicílios particulares permanentes, estimada para as áreas tipicamente rurais do Brasil, em 2010, igual a 39,91 milhões de habitantes, aproximadamente $21,0 \%$ da sua população residente. De acordo com o Censo Demográfico, em 2010, 29,54 milhões de habitantes brasileiros eram considerados rurais, representando $15,6 \%$ do total (PSBR, 2019).
} 
A Agência Nacional de Águas (ANA), por meio da publicação do relatório "Atlas Esgotos: Despoluição de Bacias Hidrográficas" (ANA, 2017), disponibilizou as informações mais recentes a respeito do saneamento em sedes municipais urbanas do Brasil. Os resultados apresentados no referido relatório indicaram que o déficit nos serviços de coleta e tratamento de esgotos no Brasil tem resultado no lançamento diário de 5.516 t de DBO nos corpos de água, o que corresponde a $60,6 \%$ da carga orgânica gerada diariamente e resulta em implicações negativas aos usos múltiplos dos recursos hídricos (ANA, 2017).

A precariedade nos serviços de esgotamento sanitário é mais acentuada em regiões periurbanas e rurais, caracterizadas por estarem situadas a longas distâncias das áreas centrais e apresentarem baixa densidade demográfica. Tais características dificultam a utilização dos sistemas centralizados de tratamento de esgotos comumente adotados em regiões urbanas e densamente povoadas (Tonetti et al., 2018).

Libralato et al. (2012) observam que, para o atendimento de regiões dispersas, a utilização de sistemas descentralizados de tratamento de efluentes tem sido apontada como uma alternativa efetiva, capaz de aumentar os índices de tratamento de esgotos nestas regiões. Isso é possível tendo em vista que sistemas descentralizados, usualmente, possuem baixo custo de implantação e operação, baixo requisito de energia/área, além de simplicidade operacional (Massoud et al., 2009).

De acordo com Libralato et al. (2012), o debate internacional evidenciou a existência de diversas restrições de natureza ambiental, social e econômica na dicotomia centralização/descentralização. Desse modo, segundo os autores, não é concebível aceitar ou recusar uma delas por pressuposição, isto é, sem a devida avaliação técnica prévia caso a caso. $\mathrm{O}$ processo de avaliação e seleção de sistemas centralizados ou descentralizados para o tratamento de esgotos em uma localidade envolve diversos fatores, tais como: a concepção do sistema de tratamento, nível de tratamento exigido, características da população atendida, custos relativos à construção, operação e manutenção dos sistemas, bem como dos custos advindos da reparação e substituição do sistema (Massoud et al., 2009).

Muitos sistemas descentralizados não fornecem o nível de tratamento apropriado à proteção da saúde pública e do ambiente. Isso ocorre em razão da ausência de programas efetivos de gerenciamento, responsáveis por assegurar a inspeção, monitoramento e manutenção regulares dos sistemas (Massoud et al., 2009).

$\mathrm{Na}$ União Europeia, os requisitos gerais relativos à instalação, operação e manutenção de pequenas unidades de tratamento de águas residuárias são definidos por meio da Norma Europeia 12.566, que contempla um conjunto de normas europeias (EN) publicadas entre os anos 2000 e 2016. Nos Estados Unidos, a Agência de Proteção Ambiental (USEPA) possui um manual com informações referentes ao uso de sistemas de tratamento de águas residuárias no local (onsite) (USEPA, 2005). Em países em desenvolvimento, ainda é verificada grande dificuldade para a manutenção dos sistemas descentralizados implementados. Para Cookey et al. 
(2016), parte das falhas verificadas nesses países pode estar associada à ausência de regulamentações ou à existência de regulamentações pouco claras.

No Brasil, a ausência de normatização e de uma clara definição legal da responsabilidade de gestão de sistemas descentralizados tende a criar setores descobertos e prejudica a implementação bem-sucedida de tais sistemas. Importante observar que com a aprovação da Lei 14.026/2020, foi incluído o seguinte parágrafo na Lei 11.445/2007, que estabelece a Política Nacional do Saneamento Básico: nas Zonas Especiais de Interesse Social (Zeis) ou outras áreas do perímetro urbano ocupadas predominantemente por população de baixa renda, o serviço público de esgotamento sanitário, realizado diretamente pelo titular ou por concessionário, inclui conjuntos sanitários para as residências e solução para a destinação de efluentes, quando inexistentes, assegurada compatibilidade com as diretrizes da política municipal de regularização fundiária. Como trata-se de uma alteração recente, ainda não é possível analisar os impactos da alteração do marco legal.

Adicionalmente, estudos que abordam a dicotomia centralização/descentralização no contexto brasileiro são escassos. A ausência de estudos que contemplam soluções descentralizadas de tratamento de esgotos pode estar associada ao estigma vinculado aos sistemas descentralizados. Segundo Tonetti et al. (2018), os sistemas descentralizados são, por vezes, considerados inferiores às soluções centralizadas adotadas em grandes centros urbanos e sinônimos de precariedade e subdesenvolvimento. No entanto, tal percepção não considera que soluções descentralizadas podem ser mais adequadas em determinados contextos.
Dentre os estudos existentes sobre essa temática, destaca-se o trabalho de Paffrath \& Freira (2019) que compararam a utilização de sistemas centralizados e descentralizados, em um município brasileiro. Outros autores, tais como Machado et al. (2017), Cruz et al. (2018) e Cruz et al. (2019) avaliaram questões associadas ao desempenho individual de sistemas de tratamento aplicados ao contexto descentralizado. No âmbito internacional, Libralato et al. (2012) apresentaram um panorama do papel da centralização e descentralização no gerenciamento de águas residuárias.

Contudo, não são verificados estudos que realizaram uma avaliação das questões associadas à utilização de sistemas descentralizados nas diferentes regiões brasileiras, tampouco que utilizaram dados atuais do cenário do saneamento brasileiro como o Atlas esgotos (ANA, 2017) e o PSBR (2019) para uma avaliação atual do saneamento descentralizado no Brasil.

Diante das lacunas, buscou-se por meio desse estudo analisar aspectos relativos à utilização de sistemas descentralizados e discutir questões relacionadas às tecnologias utilizadas e à gestão desses sistemas à luz da legislação vigente, a fim de identificar potenciais e limitações para o uso da técnica.

\section{Fundamentação teórica}

O processo de avaliação e seleção de um sistema de tratamento de esgoto mais apropriado para uma localidade envolve diversos fatores, tais como: características da população atendida e custos relativos à construção, operação e manutenção dos sistemas (Massoud et al., 2009). Além disso, é de 
fundamental importância a avaliação do sistema de gestão de efluentes mais apropriado para cada contexto. Os sistemas existentes para o tratamento de esgotos sanitários, basicamente, incluem duas abordagens: centralizadas e descentralizadas, conforme descrito a seguir (Tonetti et al., 2018).

\subsection{Sistemas centralizados}

Os sistemas centralizados têm sido empregados no tratamento de efluentes oriundos de regiões urbanizadas e com elevada densidade populacional. Tais sistemas, comumente, são de propriedade pública e realizam a coleta de grandes volumes de águas residuárias que são tratadas e descartadas distantes da fonte de geração (Libralato et al., 2012). Em função da elevada distância entre os pontos de geração e de tratamento dos efluentes em modelos de gestão centralizada, a maior parte dos custos advindos dessa concepção está associada à implantação de redes coletoras de esgotos. Segundo Zaharia (2017), tais custos representam mais de $60 \%$ do orçamento total requerido para a implantação dos sistemas.

Os sistemas centralizados são amplamente utilizados por serem considerados uma alternativa consolidada para o tratamento eficaz de esgotos. No entanto, os custos de implantação de uma malha de coleta e transporte dos esgotos de regiões periurbanas até as ETEs centralizadas, por vezes, tornam essa alternativa inviável em tais regiões (Oliveira Júnior, 2013). O menor número de beneficiários nessas áreas ocasiona perdas de economias de escala e de densidade, acarretando maiores custos com a provisão dos serviços de esgotamento sanitário (Eggimann et al., 2016). Tal fato resulta em um elevado número de pessoas sem acesso aos serviços de esgotamento sanitário, uma vez que, em países em desenvolvi- mento, a economia é o critério mais importante na tomada de decisão (Massoud et al., 2009).

\subsection{Sistemas descentralizados}

Os sistemas de gerenciamento descentralizado são definidos como aqueles cuja coleta, tratamento e disposição/reutilização de esgotos são realizadas próximas à fonte de geração. A abordagem descentralizada comumente compreende sistemas locais ou em cluster (Libralato et al., 2012; USEPA, 2005) (Figura 1).

Os sistemas locais caracterizam-se por realizar o tratamento de efluentes gerados em residências unifamiliares. Tais sistemas podem ser aplicados com ou sem separação de efluentes. Essa prática, difundida no continente europeu, é adotada em virtude da possibilidade de recuperação dos recursos potenciais advindos dos esgotos domésticos. No Brasil, usualmente, os efluentes são tratados sem separação prévia em sistemas locais de tratamento de esgotos (Massoud et al., 2009; Oliveira Júnior, 2013).

Os sistemas de tratamento comunitários (clusters), por sua vez, realizam o tratamento de efluentes de um grupo de edificações em área próxima do ponto de geração (USEPA, 2005). Não há na literatura uma definição clara do número máximo de habitantes que uma ETE descentralizada em cluster pode atender. No entanto, Massoud et al. (2009) citam que tais sistemas servem mais do que um agregado familiar, podendo atender até números superiores a cem residências. Reymond et al. (2018), por sua vez, citam que esses sistemas de tratamento de pequena escala atendem a populações de até 5.000 habitantes. 


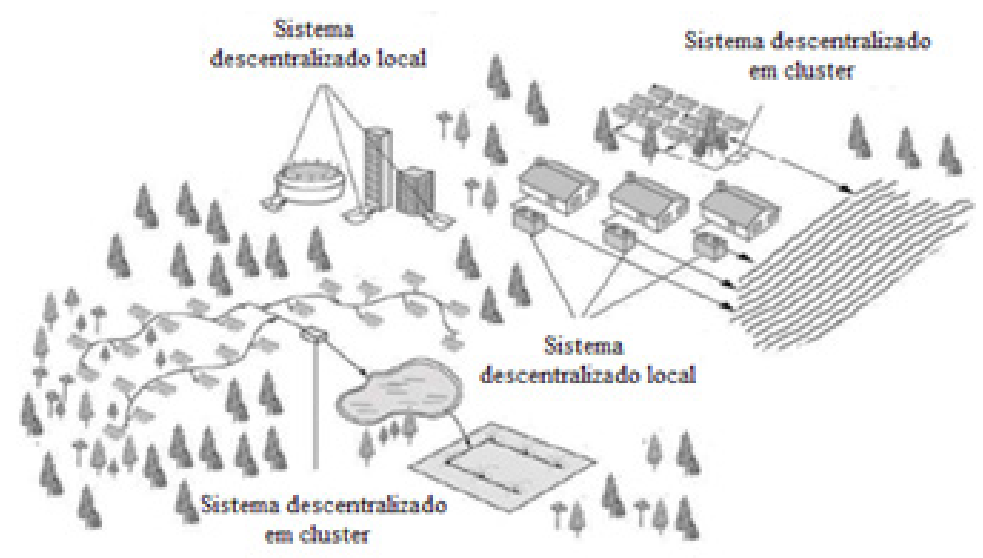

FIGURA 1 - Representação de sistemas descentralizados locais e em clusters.

FONTE: Adaptada de Asano et al. (2007).

No Brasil, o enquadramento das ETEs descentralizadas tem sido, comumente, realizado em conformidade com a legislação CONAMA ${ }^{\circ}$ 377/2006, que dispõe sobre a classificação dos sistemas em função de seus portes. Segundo a referida legislação, são consideradas de pequeno porte ETEs projetadas para atender vazões de até $50 \mathrm{~L} \mathrm{~s}^{-1}$ ou com capacidade para atendimento de população igual ou inferior a 30.000 habitantes. No entanto, segundo Orth (2007), não há uma relação direta entre ETEs descentralizadas e ETEs de pequeno porte. Segundo o autor, a classificação de uma ETE como descentralizada está relacionada a uma questão de escala, por exemplo, da comparação da população atendida pelo sistema descentralizado com a população total de um município.

A abordagem descentralizada para o tratamento de águas residuárias é citada como uma solução para a problemática do esgotamento sanitário em comunidades rurais e periurbanas, as quais não são servidas pelo sistema de esgotamento sanitário centralizado (Santos, 2019). Estudos indicam que a implantação de unidades de tratamento descentralizadas em pequenas comunidades, comparativamente às alternativas centralizadas, resulta em menores custos de implantação e operação (Eggimann et al., 2016; Jung et al., 2018). No entanto, para que os sistemas descentralizados sejam efetivos na diminuição do potencial poluidor dos esgotos sanitários, é necessário que seja implementado um programa de gestão rigoroso, que garanta a inspeção regular e a manutenção apropriada dos sistemas (Massoud et al., 2009).

Ademais, em sistemas descentralizados, o reúso não potável das águas residuárias tratadas é fortemente encorajado, sempre que possível e apropriado (Libralato et al., 2012) explicam que, na abordagem descentralizada, a utilização de sistemas em pequena escala facilita a reutilização dos efluentes no local, representando economia para as residências atendidas. Um estudo realizado por Morato et al. (2019), visando estimar a redução de consumo de água com o reúso de água cinza, indicou que haveria uma economia de água potável de cerca de $28 \%$ no consumo total de uma residência. 
Os benefícios do reúso de água em edificações que utilizam sistemas descentralizados também são descritos na literatura internacional, tais como em Opher \& Friedler (2016) e Yerri \& Piratla (2019). No entanto, no Brasil, a experiência de reúso de efluentes provenientes de ETEs descentralizadas ou oriundos de águas de lavagem das edificações ainda é incipiente.

A ausência de uma definição clara dos padrões de reúso não potável tem sido apontada como um dos fatores responsáveis pela restrita adoção da referida técnica. Desse modo, Fiori et al. (2006) destacam que, para a ampliação do reúso de água no Brasil, cabe aos órgãos competentes institucionalizar, regulamentar e promover a prática no país.

Nesse sentido, a Lei $n^{\circ}$ 14.026/2020 atualizou o marco legal do saneamento básico e alterou a Lei $n^{0} 9.984 / 2000$, estabelecendo que caberá à ANA estabelecer normas de referência sobre reúso dos efluentes sanitários tratados, em conformidade com as normas ambientais e de saúde pública. A Lei ${ }^{\circ}$ 14.026/2020 realizou, ainda, alterações na Lei $n^{\circ}$ $11.445 / 2007$, que passou a conter menções ao reúso de efluentes sanitários.

$\mathrm{Na}$ versão atual da Lei n ${ }^{\circ} 11.445 / 2007$, o fomento ao reúso de efluentes sanitários é considerado um dos princípios fundamentais da prestação de serviços públicos de saneamento básico. No conceito de esgotamento sanitário também foi realizada a inclusão de atividades relativas à destinação final dos esgotos sanitários para produção de água de reúso. $\mathrm{O}$ reúso da água também foi mencionado em relação aos contratos de prestação dos serviços públicos de saneamento básico, que deverão conter metas do reúso de efluentes e possíveis fontes de receitas alternativas, incluindo, o uso de efluentes sanitários para a produção de água de reúso. Por fim, a atualização da Lei n ${ }^{0}$ 11.445/2007 determina que edificações para uso não residencial ou condomínios, desde que autorizados pelo órgão gestor competente, poderão utilizar fontes e métodos alternativos de abastecimento de água, incluindo o reúso.

Adicionalmente, nos estados de Minas Gerais, São Paulo e Ceará, o reúso de água não potável foi objeto de regulamentação por meio da Deliberação Normativa $n^{\circ} 65 / 2020$, Resolução Conjunta SES/ $\mathrm{SMA}_{\text {SSRH }}{ }^{\circ}$ 01/2017 e Lei $\mathrm{n}^{\circ} 16.033 / 2016$, respectivamente.

\section{Método de pesquisa}

Para estruturar essa revisão foram abordados os principais aspectos relativos à utilização de sistemas centralizados e descentralizados no tratamento de esgotos sanitários. Em um primeiro momento, a partir do levantamento das características dos referidos sistemas, e da consulta à literatura técnica relacionada ao tema, fez-se a inferência de conjunturas as quais a adoção dos sistemas descentralizados é mais conveniente. Em seguida, foi realizada uma avaliação acerca dos aspectos legais relativos à utilização de sistemas descentralizados de tratamento de esgotos no Brasil. Por fim, o estudo das principais alternativas de tratamento utilizadas no saneamento descentralizado foi conduzido, tendo como foco os sistemas mais utilizados na realidade brasileira.

\subsection{Sistemas centralizados e descentralizados de tratamento de esgotos}

A base metodológica para a execução desse estudo consistiu em um levantamento bibliográfico utilizando-se a plataforma de busca do Portal de pe- 
riódicos da CAPES. Após a identificação da base de dados a ser consultada, foram definidas as palavras-chave a serem utilizadas na busca, sendo elas "Decentralized wastewater management", "Centralized wastewater management" e "Decentralization". Foram analisados, predominantemente, periódicos cuja classificação pelo sistema Qualis Periódicos da CAPES variava entre A1, A2 e B1. Após a busca, foram selecionados 36 artigos que abordavam o uso de sistemas centralizados e descentralizados. A seleção dos artigos baseou-se na existência, em seus conteúdos, de informações referentes às definições e características de tais sistemas, bem como de vantagens e desvantagens associadas ao uso de cada um deles. Adicionalmente, foram avaliados livros e manuais internacionais publicados pela Agência de Proteção Ambiental dos Estados Unidos (USEPA) e International Water Association (IWA) e livros nacionais, tendo em vista a necessidade de obtenção de informações a respeito das origens e das características dos sistemas descentralizados utilizados no Brasil. Após a realização do levantamento bibliográfico, as informações obtidas foram compiladas e relacionadas baseando-se nos diferentes enfoques dados pelos autores.

\subsection{Avaliação dos aspectos legais na utilização de sistemas descentralizados no Brasil}

A fim de identificar as limitações e potenciais no aparato legal existente no Brasil, o conceito e a utilização do saneamento descentralizado foram avaliados à luz da legislação vigente. A análise foi conduzida, individualmente, para sistemas individuais e coletivos.
O método utilizado para a obtenção das informações consistiu em uma análise documental das diretrizes que normatizam o saneamento brasileiro. A avaliação foi realizada abrangendo as regulamentações federais e estaduais existentes relativas ao tema, utilizando-se as bases de consulta de legislação do Governo Federal (Brasil, 2019). Em um primeiro momento, foram avaliadas as legislações que faziam menção ao saneamento de modo geral, realizando-se a busca no portal por meio das palavras-chave "saneamento", "esgoto" e "esgotamento". Após a seleção das legislações, foi realizada uma avaliação individual dos documentos buscando-se menções ao saneamento descentralizado, por meio de soluções individuais ou em clusters. Nesse ponto, também foi verificada a existência de menções à atribuição da responsabilidade pela implantação e gestão dos sistemas descentralizados em locais não atendidos pelas redes públicas de coleta.

A consulta das legislações vigentes foi realizada respeitando-se a hierarquia legal, com a avaliação das diretrizes constitucionais e, em seguida, das infraconstitucionais (leis, decretos, atos normativos, portarias e resoluções). Como critério de avaliação das legislações, somente documentos em vigência no ano de 2019 foram avaliados.

\subsection{Avaliação das alternativas de tratamento utilizadas no saneamento descentralizado}

Uma investigação das principais alternativas tecnológicas para o tratamento descentralizado de esgotos foi conduzida, dando ênfase àquelas citadas como mais utilizadas no país e às soluções individuais de tratamento recomendadas na NBR 13.969/1997 e no PSBR (2019). 
A fim de verificar a representatividade das alternativas tecnológicas levantadas no setor de saneamento brasileiro, foi realizada uma consulta aos dados de ETEs em operação no país, disponibilizados pela Agência Nacional de Águas e Saneamento Básico (ANA, 2017). Nessa etapa, tendo em vista a ampla utilização de tanques sépticos e filtros anaeróbios (TS+FAN) no país, foi realizada uma avaliação da existência de leis nos estados brasileiros referentes à utilização de tais sistemas. A avaliação das legislações com referência aos sistemas TS+FAN ocorreu de modo similar à citada no tópico anterior, com a realização de buscas no portal de pesquisa de legislação do Governo Federal (Brasil, 2019).

\section{Resultados e discussão}

A avaliação dos estudos que envolvem a análise de sistemas centralizados e descentralizados em diferentes contextos possibilita a identificação de vantagens e desvantagens desses sistemas. Um resumo das principais vantagens e limitações, considerando questões sociais, econômicas e ambientais é apresentado na Tabela 1.

Sobre os custos, os estudos indicam que em sistemas centralizados, os maiores custos são gerados pelo sistema de coleta e, inversamente, em sistemas descentralizados, a maior parte do investimento necessário é direcionado para a implantação da tecnologia de tratamento (Libralato et al., 2012).

Diante das vantagens e desvantagens descritas dos sistemas centralizados e descentralizados e a partir dos critérios definidos por Asano et al. (2007), pode-se inferir algumas situações as quais a adoção de sistemas descentralizados é mais indicada:
- Quando a população está localizada em regiões rurais, periurbanas ou de baixa densidade demográfica;

- Quando a comunidade, condomínio ou conjunto habitacional está localizada distante de um sistema de coleta e tratamento de esgotos já existente;

- Quando existem oportunidades e demandas locais de reúso de água;

- Quando os sistemas centralizados existentes não possuem capacidade de atender toda a população e os recursos para expansão são limitados.

Adicionalmente, os sistemas descentralizados são indicados para o atendimento de comunidades isoladas situadas em territórios especiais, tais como unidades de conservação, territórios quilombolas, terras indígenas e demais territórios habitados por populações tradicionais. De acordo com Tonetti et al. (2018), tais territórios possuem especificidades que os diferenciam dos núcleos atendidos por sistemas centralizados de saneamento, desse modo, requerem abordagens diferentes para a implantação e operação dos seus sistemas de saneamento.

Santos (2019) cita que, em regiões metropolitanas, os sistemas descentralizados têm sido a opção preferencial para o atendimento de zonas periféricas, vilas, conjuntos habitacionais distantes e áreas de baixa renda, que se caracterizam como sistemas isolados não interligados aos sistemas centralizados de maior porte. Contudo, o autor ressalta que excessos de descentralização podem resultar na existência de muitos microssistemas pulverizados, o que pode multiplicar as demandas de manejo e gestão. 
TABELA 1 - Principais vantagens e desvantagens associadas à utilização de sistemas de tratamento de esgotos centralizados e descentralizados.

\section{Sistemas centralizados}

\section{Vantagens}

Economias de escala em áreas densamente povoadas

Elevada aceitação social

Regulamentação consolidada

Múltiplos níveis de qualidade de água possíveis

Normalmente possui unidades para processamento da fase sólida

Variações de vazão e carga são absorvidas pelas extensas redes coletoras

Tratamento em níveis seguros para a saúde pública e do corpo receptor

\section{Desvantagens}

Baixo atendimento às populações periurbanas e rurais

Alto custo de implantação

Operação e manutenção normalmente complexas

Grande volume de efluente descartado pode resultar em eutrofização

Forte dependência do fornecimento de energia elétrica

Deseconomias de escala são possíveis no atendimento de longas distâncias

Necessidade de renovação do sistema de coleta a cada 50-60 anos

\section{Sistemas descentralizados}

\begin{tabular}{|c|c|}
\hline Vantagens & Desvantagens \\
\hline Atende áreas periurbanas, rurais e de baixa densidade populacional & $\begin{array}{l}\text { Manutenção inapropriada deixa o sistema altamente } \\
\text { exposto a falhas }\end{array}$ \\
\hline Requisitos simples de operação e manutenção & Ausência de regulamentação consolidada \\
\hline Potencial reutilização de águas residuárias tratadas & $\begin{array}{l}\text { Instalação e gerenciamento de diversos sistemas podem ser } \\
\text { difíceis }\end{array}$ \\
\hline Possibilidade de construção gradativa do sistema (evita capacidade ociosa) & Ampla variação de vazão e carga poluente no esgoto \\
\hline \multicolumn{2}{|l|}{ Promove o retorno das águas residuárias tratadas dentro da bacia de origem } \\
\hline \multicolumn{2}{|l|}{ Tratamento em níveis seguros para a saúde pública e do corpo receptor } \\
\hline
\end{tabular}

FONTE: Libralato et al. (2012); Massoud et al. (2009); Santos (2019); Tchobanoglous et al. (2004); Tonetti et al. (2018).

Por outro lado, regiões com elevada densidade populacional ou elevado nível de urbanização, onde são permitidos ganhos em escala, favorecem o uso de sistemas centralizados. No entanto, quando existem elevadas densidades populacionais em áreas periurbanas localizadas a grandes distâncias dos sistemas centralizados, essa economia de escala desaparece, exigindo elevado investimento (Subtil et al., 2016).
Desse modo, percebe-se que a abordagem descentralizada não está em contraste com a centralizada. A descentralização é uma alternativa para integrar os sistemas convencionais existentes. Orth (2007) observa que ambas configurações de sistemas são aplicáveis em diferentes situações, cabendo aos gestores do setor a decisão do sistema que melhor se aplica aos diferentes contextos. Assim, verifica-se que a definição do sistema ideal 
a ser implementado em uma área está vinculada a uma profunda análise das características regionais, da avaliação das instalações já existentes e de demandas específicas da população atendida.

Tonetti et al. (2018) ressaltam que é fundamental que sejam adotadas estratégias diversas, que considerem as peculiaridades locais e regionais e respeitem a diversidade cultural e ambiental das comunidades. Desse modo, não há uma solução única que possa ser aplicada a todas as situações. Propostas apropriadas consistem em adotar a coexistência de diversos níveis de centralização e descentralização, explorando as potencialidades que cada sistema oferece. A abordagem descentralizada, por exemplo, mostra-se muito aplicável no caso de grandes blocos, tais como hospitais, centros comerciais e aeroportos. Por outro lado, em áreas urbanas densamente povoadas e historicamente servidas por sistemas centralizados, a descentralização não representa uma alternativa viável (Libralato et al., 2012).

\subsection{Sistemas descentralizados e aspectos legais}

A Constituição Federal brasileira, promulgada no ano de 1988, estabelece que a saúde e o meio ambiente equilibrado são direitos fundamentais de todo cidadão. Segundo Freeman et al. (2017), o saneamento deficiente resulta na incidência de diversas doenças e, desse modo, apresenta relação direta com as questões de saúde pública. Diante disso, verifica-se que na ausência de condições sanitárias apropriadas, o direito à saúde e ao meio ambiente equilibrado, previstos constitucionalmente, não pode ser efetivado.
As linhas gerais, instituídas na Constituição Federal, delinearam o desenvolvimento das diretrizes infraconstitucionais para o saneamento existentes atualmente no Brasil. Destas, a Política Federal de Saneamento Básico (PFSB), com diretrizes instituídas pela Lei Federal 11.445/2007, é considerada a mais importante na conjuntura nacional do saneamento básico. A referida Lei, dentre suas atribuições, estabelece os princípios para a provisão do saneamento e determina a elaboração do Plano Nacional de Saneamento Básico (Plansab). Por meio do Decreto Federal 7.217/2010, a regulamentação da PFSB foi efetivada. Adicionalmente, o referido Decreto apresentou complementações às disposições da Lei 11.445/2007 ao definir como obrigatória a utilização dos serviços públicos de saneamento e ao sujeitar a execução dos planos e projetos de saneamento ao licenciamento ambiental.

Segundo dados do Plansab, a maior parcela do déficit de saneamento básico no Brasil é verificada em áreas rurais e periferias urbanas. Em função disso, o plano manifestou a necessidade de elaboração de um programa de saneamento rural que contemple essa população. Neste contexto, foi aprovado, em dezembro de 2019, o Programa Saneamento Brasil Rural (PSBR), sob coordenação do Ministério da Saúde.

As informações contidas no PSBR revelaram que apenas $20,6 \%$ da população residente em áreas rurais brasileiras possuem atendimento apropriado ao esgotamento sanitário, sendo os piores índices verificados em aglomerações isoladas menos adensadas e em locais sem aglomerações. Ademais, a discussão do atendimento descentralizado, com a provisão destes serviços em regiões dispersas, é recente na legislação federal. A Lei n ${ }^{\circ}$ 14.026/2020, que atualizou o marco legal do saneamento básico 
e alterou a Lei $n^{\circ} 11.445 / 2007$, estabeleceu que é facultado à entidade reguladora prever hipóteses em que o prestador de serviços de saneamento poderá utilizar métodos alternativos e descentralizados para os serviços de coleta e tratamento de esgoto em áreas rurais, remotas ou em núcleos urbanos informais consolidados.

No nível estadual, ainda que de modo incipiente, são verificadas legislações que dispõem de diretrizes a respeito da utilização de sistemas de tratamento de esgotos em áreas não atendidas pelos serviços públicos, cujos detalhes serão abordados nos tópicos seguintes.

\subsubsection{Sistemas locais}

ALei Federal no 11.445/2007 determina que, na ausência de redes públicas de saneamento, soluções individuais de afastamento e tratamento de esgotos são admitidas. No Brasil, os sistemas descentralizados locais (ou individuais) comumente utilizam tanques sépticos (TS) como opção de tratamento, com disposição final nos solos, sumidouros ou corpos hídricos (Oliveira Júnior, 2013). Segundo o mesmo autor, tais sistemas, em função de uma inapropriada rotina de descarte de sólidos, por vezes, não apresentam efluentes com a qualidade necessária para proteção dos cursos de água e da saúde pública. A falta de manutenção em tais unidades, pode estar associada à ausência de uma regulamentação específica, que defina com clareza as responsabilidades sobre a manutenção dos sistemas e a disposição final dos resíduos (ANA, 2017).

A Lei Federal 11.445/2007 determina que "não constitui serviço público a ação de saneamento executada por meio de soluções individuais, desde que o usuário não dependa de terceiros para operar os serviços (...)". O Decreto $n^{\circ} 7.217 / 2010$, por sua vez, cita que serão considerados serviços públicos "a fossa séptica e outras soluções individuais de esgotamento sanitário, quando se atribua ao Poder Público a responsabilidade por sua operação, nos termos de norma específica". A falta de clareza quanto às atribuições faz com que o gerador e o poder público, por vezes, não assumam a responsabilidade pelo manejo de resíduos dos sistemas, o que resulta em falhas operacionais.

Nas Zonas Especiais de Interesse Social (ZEIS) e demais áreas do perímetro urbano ocupadas predominantemente por população de baixa renda, a Lei $\mathrm{n}^{\circ} 14.026 / 2020$, que atualizou a Lei $\mathrm{n}^{\mathrm{o}} 11.445 / 2007$, atribui as ações de esgotamento sanitário ao serviço público, que deve incluir conjuntos sanitários para as residências e solução para a destinação de efluentes, quando inexistentes. Tal proposição não especifica se a utilização, quando necessária, de sistemas alternativos de esgotamento sanitário pelo serviço público deverá ser realizada por meio de sistemas individuais ou coletivos.

Além dos entraves relacionados à responsabilidade pela manutenção dos sistemas individuais, o destino do lodo proveniente de tais unidades também constitui um problema. Segundo Andreoli (2009), o lodo é um passivo, assim, historicamente, há uma grande resistência do poder público e da população em assumir a responsabilidade por sua gestão.

De acordo com o Decreto $n^{\circ} 7.217 / 2010$, considera-se serviço público de esgotamento sanitário "a disposição final dos esgotos sanitários e dos lodos originários da operação de unidades de tratamento coletivas ou individuais, inclusive fossas sépticas". A Lei ${ }^{\circ}$ 14.026/2020 incluiu, ainda, que a disposição deverá ser realizada de forma ambientalmente apropriada. 
Atualmente, verifica-se que a gestão do lodo gerado em sistemas individuais no país divide-se entre o poder público, por meio das prefeituras municipais, e empresas privadas que oferecem esse serviço. Algumas dessas empresas não são licenciadas e, muitas vezes, não fornecem uma apropriada destinação ao material recolhido (Andrade et al., 2017). A inapropriada destinação do material coletado em tanques sépticos pode resultar na contaminação dos corpos hídricos e representar riscos à saúde pública e ao ambiente. Por esse motivo, a gestão apropriada do lodo proveniente de tanques sépticos é uma necessidade crítica

Nos Estados Unidos, a gestão do lodo oriundo de sistemas individuais é realizada baseando-se nas recomendações de tecnologias para o tratamento e descarte do lodo da Agência de Proteção Ambiental (USEPA, 1999). Contudo, em países em desenvolvimento, tais como Brasil, Índia (Prasad \& Ray, 2019) e Camboja (Frenoux \& Tsitsikalis, 2015), o descarte e o tratamento do lodo séptico ainda são problemas de primeira ordem.

No Brasil, apenas o estado de Santa Catarina possui legislação que define as responsabilidades pelos sistemas individuais de tratamento (Tabela 2). Nos estados do Rio de Janeiro e Amazonas são verificadas leis que possuem foco no destino do lodo após coleta, apresentando mecanismos de controle e fiscalização dos caminhões "limpa-fossa".

A utilização de dispositivos de rastreamento nos caminhões que realizam o esgotamento de tanques sépticos é considerada uma alternativa para fiscalização do correto descarte do lodo coletado. Andreoli (2009) cita que os sistemas de informação geográfica (SIG) são importantes ferramentas para fiscalização e monitoramento de atividades relativas à gestão do lodo de tanques sépticos. $\mathrm{O}$ autor menciona, ainda, a necessidade de obtenção de informações prévias acerca das atividades de coleta e transporte de lodo podendo, para isso, serem instalados equipamentos de GPS nos caminhões "limpa-fossa", que facilitarão o acompanhamento de suas rotas. Os estados do Amazonas (Resolução CEMAN no 27/2017), Santa Catarina (Lei $n^{\circ}$ 17.082/2017) e Rio de Janeiro (Lei n ${ }^{\circ} 17.082 / 2017$ ) são pioneiros na criação de leis que determinam a utilização dessa tecnologia.

\subsubsection{Sistemas em clusters}

De acordo com as disposições da Resolução CONAMA n ${ }^{\circ}$ 430/2011, a implantação de sistemas de tratamento de esgotos em locais como condo-

TABELA 2 - Regulamentações referentes à gestão do lodo em sistemas descentralizados em estados brasileiros.

\begin{tabular}{|c|c|c|}
\hline \multicolumn{3}{|r|}{ Esfera estadual } \\
\hline Estado & Legislação & Enfoque \\
\hline Amazonas & $\begin{array}{l}\text { Resolução CE- } \\
\text { MAN n }{ }^{\circ} 27 / 2017\end{array}$ & $\begin{array}{l}\text { Regulamenta a obrigatoriedade da instalação de sistema de rastreamento via satélite nos } \\
\text { veículos prestadores de serviços de coleta de resíduos doméstico/sanitários. }\end{array}$ \\
\hline Rio de Janeiro & Lei n ${ }^{\circ} 6.862 / 2014$ & $\begin{array}{l}\text { Determina que os veículos responsáveis pela limpeza de tanques sépticos devem ser equipa- } \\
\text { dos com rastreador. }\end{array}$ \\
\hline Santa Catarina & Lei n ${ }^{\circ} 17.082 / 2017$ & Obriga os caminhões limpa fossa a instalarem dispositivo de geoposicionamento. \\
\hline Santa Catarina & Lei $n^{\circ} 17.492 / 2018$ & $\begin{array}{l}\text { Define que a instalação, funcionamento e manutenção de sistemas individuais ficarão a } \\
\text { cargo do proprietário em todo o estado. }\end{array}$ \\
\hline
\end{tabular}


mínios, conjuntos habitacionais e pequenas comunidades, requer autorização do órgão ambiental competente, o qual cabe a função de classificar o empreendimento quanto ao seu potencial poluidor e, a partir disso, definir os requisitos necessários para o licenciamento ambiental, quando necessário.

Assim como em sistemas centralizados, ETEs descentralizadas devem atender aos requisitos dispostos em legislação, referentes a normas de projeto e atendimento aos padrões de lançamento de efluentes em corpos hídricos. No Brasil, tais padrões estão dispostos na Resolução CONAMA no 430/2011. Em suas diretrizes, a Resolução estabelece que os responsáveis por fontes poluidoras devem realizar o automonitoramento dos efluentes lançados em corpos hídricos, sendo dispensadas fontes de baixo potencial poluidor.

Para a implantação de um grupo de domicílios em áreas não atendidas por serviços públicos de esgotamento sanitário, cabe ao empreendedor a busca pela solução técnica mais apropriada para a coleta e tratamento dos esgotos gerados. O mesmo procedimento deve ser realizado para a implantação de moradias de interesse social, conforme estabelecido na Lei ${ }^{\circ}$ 11.977/2009 e na Resolução CONAMA n ${ }^{\circ}$ 412/2009.

Após a implantação das ETEs descentralizadas, a responsabilidade pela operação e manutenção dos sistemas de tratamento não é definida em regulamentações a nível federal. Contudo, alguns estados definem a responsabilidade pela manutenção e operação desses sistemas após a construção. Em Santa Catarina, a Lei n ${ }^{\circ}$ 17.492/2018 determina que, havendo a necessidade de implantação de ETEs descentralizadas, fica a cargo da concessionária de saneamento a operação, manutenção e exploração comercial da ETE. No estado do Maranhão, por sua vez, por meio da Lei $n^{\circ} 9.550 / 2012$, foi determinado que edifícios que possuem mais de três andares e condomínios que contém mais de 10 unidades devem ter, obrigatoriamente, pequenas ETEs. A referida Lei estabelece ainda que a operação e a manutenção das ETEs poderão ser realizadas pelo órgão responsável no âmbito do estado. Conforme indicado nos exemplos anteriores, soluções de tratamento de esgotos em nível comunitário são passíveis de implementação pública, podendo ser incentivadas e controladas pelos operadores dos sistemas em locais onde a implantação de redes públicas de coleta é inviável (Larsen et al., 2013).

Segundo Tchobanoglous et al. (2004), a implantação de ETEs descentralizadas só deve ser permitida mediante a designação de uma agência pública ou privada para realizar inspeções e manutenções necessárias. A proposição de Tchobanoglous et al. (2004), tem se mostrado efetiva em países que a adotaram. Langergraber et al. (2018) explicam que, na Áustria, as autoridades locais solicitam que os proprietários de ETEs possuam contrato de manutenção com uma empresa especializada ou que os proprietários realizem um curso de formação de operadores de pequenas ETEs, oferecido pelo poder público.

A implementação das medidas descritas por Tchobanoglous et al. (2004) e Langergraber et al. (2018) na elaboração de projetos de ETEs descentralizadas no Brasil poderia atuar como uma ferramenta auxiliar na gestão dos recursos hídricos. Tal suposição baseia-se no fato de que ETEs descentralizadas, quando submetidas a operação e manutenção frequentes, são capazes de fornecer efluentes com qualidade compatível com a exigida na legislação ambiental vigente (Mesquita et al., 2019). 
Garrido et al. (2016) também apontam os desafios de gestão de sistemas de saneamento instalados em áreas rurais. Segundo os autores, tais regiões, normalmente, não são atendidas pelas companhias estaduais de saneamento e, por vezes, são deixadas em segundo plano pelos departamentos ou autarquias municipais. Em consequência disso, a implantação dos sistemas de saneamento é feita pela própria comunidade, comumente, sem qualquer apoio técnico. Os autores ressaltam que, em alguns casos, há o auxílio de programas de desenvolvimento por meio de investimentos nos sistemas que, posteriormente, passam a ser gerenciados pela própria comunidade. Contudo, sabe-se que, com a ausência de acompanhamento técnico, a infraestrutura implementada pode não ser utilizada corretamente ou ser perdida em razão da ausência de manutenção.

Considerando tal vácuo institucional, alguns estados do Brasil adotaram modelos de gestão multicomunitários, que tem como objetivo garantir a operação e manutenção de sistemas de água e esgoto, em áreas rurais. De acordo com Garrido et al. (2016), neste modelo, a gestão é realizada de maneira comum para diversas comunidades dentro de uma abrangência geográfica regional. Os autores citam como exemplo dos modelos de gestão multicomunitários, a Central das Associações Comunitárias para Manutenção de Sistemas de Abastecimento de Água e Esgotos Sanitários (CENTRAL), na Bahia, e o Sistema Integrado de Saneamento Rural (SISAR), no Ceará e no Piauí. Contudo, os autores ressaltam que em uma amostra de modelos multicomunitários avaliados em sete estados brasileiros, a operação do serviço de esgoto pelos prestadores mostrou-se restrita, possuindo maior ênfase na gestão do abastecimento de água.
Segundo os autores, isso ocorre em função de no meio rural brasileiro predominar o uso de sistemas individuais (tanque sépticos), não havendo tradição de operação coletiva desse serviço.

Por fim, independente do modelo de gestão dos serviços de saneamento adotado em áreas rurais, é fundamental a avaliação da alternativa de menor esforço institucional e menor custo de implementação, operação e manutenção, a fim de assegurar que o modelo seja sustentável no longo prazo e apropriado a cada realidade local ou regional (Garrido et al., 2016).

\subsection{Alternativas tecnológicas para sistemas descentralizados}

O uso de sistemas de baixa demanda energética e simplicidade operacional em sistemas descentralizados tem se destacado em função da facilidade operacional e economia nos custos em países como Colômbia, Brasil e Índia. De modo geral, as tecnologias de tratamento utilizadas em sistemas descentralizados devem ter baixa exigência de manutenção e serem capazes de absorver as oscilações de vazão e concentração dos efluentes ao longo do dia (Massoud et al., 2009). Adicionalmente, segundo os mesmos autores, a seleção da tecnologia de tratamento a ser utilizada depende das características socioeconômicas da região e das normas vigentes. Tecnologias com alta eficiência e menor investimento global destacam-se como soluções mais viáveis em países em desenvolvimento.

Diversas opções de tecnologias para o tratamento de esgotos em sistemas descentralizados são apontadas na literatura, tais como wetlands construídos, reatores UASB, lodos ativados, lagoas de 
estabilização, filtros anaeróbios e filtros de areias (Santos, 2019). Os sistemas indicados, têm sido utilizados de modo combinado com tanques sépticos (TS) (Massoud et al., 2009). O TS é a tecnologia de tratamento mais amplamente utilizada no saneamento descentralizado em diversos países. Nos Estados Unidos, estima-se que cerca de 20 a 25\% dos domicílios são atendidos por tal sistema (Lin et al., 2017). Na Austrália, por sua vez, o saneamento rural é realizado por meio de TS, que atendem a uma população de cerca 180.000 habitantes (Buchanan et al., 2018).

No Brasil, embora não haja uma política de gestão do saneamento descentralizado, o uso de sistemas adotados mundialmente no tratamento descentralizado de esgotos é comum. Segundo Oliveira Júnior (2013), sistemas individuais de tratamento de esgoto doméstico foram implementados no Brasil inicialmente por meio da instalação de TS e tanques Imhoff em locais desprovidos de coleta e transporte de esgotos. Posteriormente, tecnologias de tratamento como filtros anaeróbios e reatores UASB foram adotadas, a fim de melhorar a qualidade do efluente tratado e, ao mesmo tempo, manter a característica de baixo custo operacional desejada para sistemas descentralizados.
Diversas alternativas tecnológicas de pós tratamento de efluentes de TS são indicadas na NBR 13.969 (ABNT, 1997). Tais tecnologias apresentam diferenças em termos de eficiência, custos, demanda de área, manutenção e operação. Desse modo, a seleção da alternativa apropriada para cada local deve basear-se na avaliação criteriosa de cada um desses fatores. Na Tabela 3 são dispostas algumas características dos sistemas citados.

Avaliando-se a Tabela 3 e levando-se em consideração os requisitos desejáveis para sistemas descentralizados (operação e manutenção simples e baixo custo operacional), os filtros anaeróbios, valas de infiltração e lagoa com plantas se destacam. Contudo, no banco de dados obtido junto à ANA (2017), referente às ETEs em operação no Brasil, não consta a presença de ETEs que utilizam valas de infiltração ou lagoa com plantas como sistemas de tratamento. A ausência dos referidos sistemas no banco de dados avaliado pode estar relacionada à maior demanda de área requerida para sua instalação, o que, por vezes, limita a sua aplicação em áreas urbanas. Adicionalmente, as informações oriundas da ANA (2017) abrangem, exclusivamente, soluções utilizadas em sedes urbanas, não sendo avaliadas aquelas adota-

TABELA 3 - Características de alternativas de pós tratamento de efluentes de tanques sépticos.

\begin{tabular}{|c|c|c|c|c|c|c|}
\hline \multirow[b]{2}{*}{ Característica } & \multicolumn{6}{|c|}{ Alternativa de tratamento } \\
\hline & Filtro anaeróbio & Filtro aeróbio & Filtro de areia & Vala de infiltração & Lodos ativados & $\begin{array}{c}\text { Lagoa com } \\
\text { plantas }\end{array}$ \\
\hline Eficiência Ssed $(\%)^{2}$ & $>70$ & $>90$ & $>90$ & $>90$ & $>90$ & $>90$ \\
\hline Área necessária & Reduzida & Reduzida & Média & Média & Média & Média \\
\hline Custo operacional & Baixo & Alto & Médio & Baixo & Alto & Baixo \\
\hline Manutenção & Simples & Simples & Simples & Simples & Complexa & Simples \\
\hline
\end{tabular}

NOTA: ${ }^{1}$ Eficiências consideradas em conjunto com o tanque séptico. ${ }^{2}$ Ssed: sólidos sedimentáveis 
das em áreas rurais, cuja disponibilidade de áreas é, comumente, maior.

Na Figura 2 são indicados os sistemas de tratamento utilizados em pequenas ETEs (população $\leq$ 2.000 habitantes), inventariadas pela ANA (2017). Verifica-se uma menor participação dos sistemas constituídos por lodos ativados. Segundo Oliveira Júnior (2013), a ausência desses sistemas em ETEs de pequeno porte está associada à necessidade de utilização de sistemas de aeração e de maior intervenção operacional, o que resulta em elevado custo de operação com energia elétrica e necessidade de contratação de operadores.

As lagoas de estabilização são o terceiro sistema mais utilizado nas ETEs brasileiras de pequeno porte. De acordo com Chernicharo et al. (2018), um dos motivos para a aplicação de lagoas em instalações de pequeno porte, é a menor demanda operacional que está, majoritariamente, associada às operações de limpeza da área. Contudo, assim como descrito para as valas de infiltração e lagoas com plantas, as lagoas de estabilização possuem alto requisito de área para sua implantação, o que pode ser um fator limitante para sua utilização em locais com limitada área disponível, tais como condomínios residenciais.

Os reatores UASB, embora não estejam presentes entre as alternativas de tratamentos citadas pela ABNT (1997), representam cerca de $40 \%$ das pequenas ETEs inventariadas. A ampla utilização de tais sistemas no saneamento descentralizado ocorreu em função da busca por soluções capazes de substituir os TS, a fim de produzir um efluente de melhor qualidade, mantendo os custos operacionais baixos (Oliveira Júnior, 2013). No entanto, sabe-se que os reatores UASB, quando comparados aos TS, demandam maior frequência de práticas operacionais, como a remoção do lodo de excesso. Desse modo, para sua implantação a nível comunitário, faz-se necessária a existência de um operador capacitado para realizar as operações necessárias para o seu bom funcionamento.

O segundo sistema mais utilizado, correspondendo a cerca de $31 \%$ das ETEs em operação de pequeno porte, é constituído por tanques sépticos seguidos por filtros anaeróbios (TS+FAN). A disseminação da utilização conjunta de tanques sépticos e filtros anaeróbios no país é atribuída, além do custo reduzido, à simplicidade de dimen-

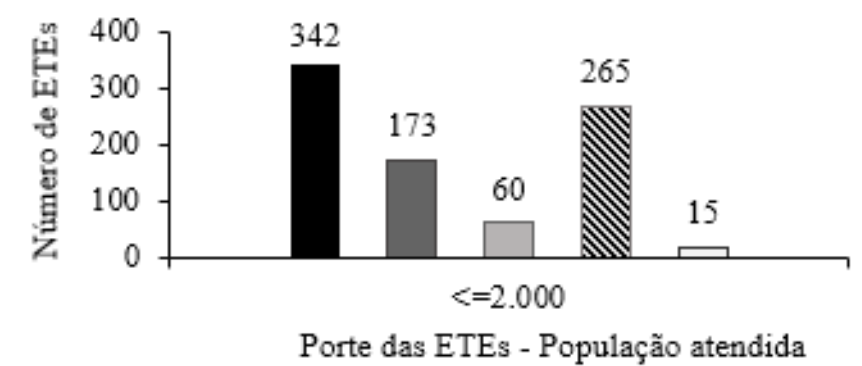

- Reator anaeróbio

- Lagoas de estabilização 口Lodos ativados s Tanque séptico + filtro anaeróbio 口Outros

Porte das ETEs - População atendida

FIGURA 2 - Sistemas de tratamento utilizados no Brasil em função do porte das ETEs. 
sionamento e construção. A partir da publicação da NBR 7.229/1993 e NBR 13.996/1997, os sistemas TS+FAN foram difundidos no país, sobretudo em sistemas descentralizados de tratamento. Isso ocorreu em função das referidas normas apresentarem informações de maneira concisa a respeito do projeto, construção e operação de tais unidades (Subtil et al., 2016). Em alguns estados brasileiros é definida a utilização de TS+FAN como opção de tratamento de esgotos, na ausência de redes públicas (Tabela 4).

TABELA 4 - Regulamentações estaduais referentes à utilização de sistemas TS+FAN.

\begin{aligned} & \hline \multicolumn{1}{c}{ Esfera estadual } \\ & \hline Estado \multicolumn{1}{c}{ Regulamentação } \\ & \hline Espírito Santo Lei $^{0} 7.499 / 2003 \\ &$ Minas Gerais $^{1}$ Lei $^{\circ} 10.793 / 1992 \\ &$ Santa Catarina Lei $^{\circ} 17.492 / 2018 \\ &$\hline\end{aligned}

${ }_{1}^{1}$ Aplicável para sistemas localizados em bacias de mananciais de abastecimento.

No que se refere ao PSBR (2019), as alternativas tecnológicas, para os sistemas descentralizados de esgotamento sanitário, foram estabelecidas levando em consideração a disponibilidade de água encanada no domicílio, bem como a profundidade do lençol freático dos solos que, eventualmente, receberão esgotos. Desse modo, foram incluídas no PSBR alternativas tecnológicas com e sem veiculação hídrica, além de alternativas que possibilitam o aproveitamento de compostos orgânicos produzidos.

No tocante aos sistemas coletivos de tratamento, foram definidas alternativas que demandam disponibilidade hídrica suficiente para o transporte dos efluentes até o local de tratamento, tais como: tanque séptico; reator UASB; reator anaeróbio compartimentado; filtro anaeróbio; lagoas de estabiliza- ção; wetlands; fertirrigação; rampa de escoamento; infiltração rápida; filtro de areia; e filtro biológico. Vale ressaltar que a utilização conjunta das referidas alternativas de tratamento foi indicada em diferentes arranjos passíveis de serem utilizados em função das condições locais.

Para os sistemas individuais, por sua vez, foram incluídas alternativas tecnológicas com e sem veiculação hídrica das excretas, a saber: fossa seca; tanque de evapotranspiração; sumidouro; fossa absorventes; tanque séptico; vala de infiltração; wetland; filtro anaeróbio; filtro de areia; círculo de bananeira; e fertirrigação subsuperficial. De maneira semelhante à citada para os sistemas coletivos, as alternativas apresentadas para os sistemas individuais foram indicadas em diversos arranjos passíveis de utilização nas áreas rurais brasileiras.

\section{Considerações finais}

O cenário atual do saneamento no Brasil aponta que, para atingir a universalização do saneamento, é necessário empreender esforços na ampliação do atendimento dos serviços de esgotamento sanitário, sobretudo, em áreas rurais e de difícil acesso à rede coletiva de coleta.

Ao se avaliar os aspectos conceituais das abordagens centralizadas e descentralizadas para o tratamento de esgotos, verifica-se que a atuação de sistemas descentralizados na matriz tecnológica do setor pode contribuir para o aumento dos índices de atendimento nas áreas anteriormente citadas. No entanto, para garantir que tais sistemas atuem de maneira efetiva na proteção da saúde pública e do ambiente, diversos desafios ainda devem ser superados. 
Foi verificado que o Brasil carece de regulamentação que defina, de maneira clara, as responsabilidades pela manutenção e operação dos sistemas descentralizados. Apenas os estados do Maranhão e Santa Catarina possuem regulamentações que definem as responsabilidades pela gestão dos sistemas descentralizados instalados. Também foram verificadas leis em vigência nos estados do Amazonas, Rio de Janeiro e Santa Catarina relacionadas à gestão de sistemas descentralizados, contudo, estas possuem foco no controle e fiscalização do destino do lodo gerado nos sistemas. Verificou-se, ainda, que entre as alternativas tecnológicas utilizadas em ETEs de pequeno porte no Brasil, os TS+FAN e os reatores UASB apresentam destaque. Três estados brasileiros, localizados no Sudeste e Sul, possuem leis que determinam a utilização de TS+FAN na ausência de redes coletivas de coleta.

Por fim, verifica-se que a adoção de sistemas descentralizados, como solução de esgotamento sanitário, pode ter maior sustentabilidade econômica e social em algumas áreas no país. Contudo, a apropriação tecnológica não deve ser entendida como ausência de operação e manutenção. De maneira oposta, os sistemas simplificados e descentralizados devem ser vistos como sistemas que, quando submetidos às práticas apropriadas de operação e manutenção, possibilitam a obtenção de efluentes com qualidade compatível com a exigida em legislação.

\section{Agradecimentos}

O presente trabalho foi realizado com apoio da Coordenação de Aperfeiçoamento de Pessoal de Nível Superior - Brasil (CAPES) - Código de Financiamento 001 e Conselho Nacional de De- senvolvimento Científico e Tecnológico (CNPq) (processo ${ }^{\circ} 131819$ / 2017-8).

\section{Referências}

ANA - Agência Nacional das Águas. Atlas esgotos: despoluição de bacias hidrográficas. Brasília: ANA, 2017.

ABNT - Associação Brasileira de Normas Técnicas. NBR 13969: Tanques sépticos - Unidades de tratamento complementar disposição final dos efluentes líquidos - Projeto, construção e operação. Rio de Janeiro: ABNT, 1997.

Andrade, C. F.; Von Sperling, M.; Manjate, E. S. Treatment of septic tank sludge in a vertical flow constructed wetland system. Engenharia Agrícola Jaboticabal, 37(4), 811-819, 2017. doi: 10.1590/1809-4430-eng.agric. v37n4p811-819/2017

Andreoli, C. V. Lodo de fossa e tanque séptico: caracterização, tecnologias de tratamento, gerenciamento e destino final. Rio de Janeiro: ABES, 2009.

Asano, T.; Burton, F. L.; Leverenz, H. L.; Tsuchihashi, R.; Tchobanoglous, G. Water Reuse: Issues, Technologies, and Applications. New York: McGraw Hill, 2007.

Brasil. Portal da legislação - governo federal, 2019. Disponível em: <http://www4.planalto.gov.br/legislacao>. Acesso em: jan. 2019.

Buchanan, N. A.; Young, P.; Cromar, N. J.; Fallow, H. J. Performance of a high rate algal pond treating septic tank effluent from a community wastewater management scheme in rural South Australia. Algal Research, 35, 325-332, 2018. doi: 10.1016/j.algal.2018.08.036

Chernicharo, C. A. L.; Ribeiro, T. B.; Garcia, G. B.; Lermontov, A.; Platzer, C. J.; Possetti, G. R. C.; Rosseto, M. A. L. L. R. Panorama do tratamento de esgoto sanitário nas regiões Sul, Sudeste e Centro-Oeste do Brasil: tecnologias mais empregadas. Revista DAE, 66(214), 5-19, 2018. doi: 10.4322/dae.2018.028

Cookey, P. E.; Koottatep, T.; Van der Steen, P.; Lens, P. N. Public health risk assessment tool: strategy to improve public policy framework for onsite wastewater treatment systems (OWTS). Journal of Water, Sanitation and Hy- 
giene for Development, 6(1), 74-88, 2016. doi: 10.2166/ washdev.2016.081

Cruz, L. M.; Tonetti, A. L.; Gomes, B. G. L. A. Association of septic tank and sand filter for wastewater treatment: full-scale feasibility for decentralized sanitation. Journal of Water, Sanitation and Hygiene for Development, 8(2), 268-277, 2018. doi: 10.2166/washdev.2018.094

Cruz, L. M. O.; Gomes, B. G. L. A.; Tonetti, A. L.; Figueiredo, I. C. S. Using coconut husks in a full-scale decentralized wastewater treatment system: The influence of an anaerobic filter on maintenance and operational conditions of a sand filter. Ecological Engineering Journal, 127, 454-459, 2019. doi: 10.1016/j.ecoleng.2018.12.021

Eggimann, S.; Truffer, B.; Maurer, M. The cost of hybrid waste water systems: A systematic framework for specifying minimum cost-connection rates. Water Research, 103, 472-484, 2016. doi: 10.1016/j.watres.2016.07.062

Fiori, S.; Fernandes, V. M. A.; Pizzo, H. Avaliação qualitativa e quantitativa do reúso de águas cinzas em edificações. Ambiente construído, 6(1), 19-30, 2006. Disponível em: https://www.seer.ufrgs.br/ambienteconstruido/article/ view/3676

Freeman, M. C.; Garn, J. V.; Sclar, G. D.; Boisson, S.; Medlicott, K.; Alexander, K. T.; Penakalapatia, G.; Anderson, D.; Mahtani, A. G.; Grimes, J. E. T.; Rehfuess, E. A.; Clasen, T. F. The impact of sanitation on infectious disease and nutritional status: A systematic review and meta-analysis. International journal of hygiene and environmental health, 220(6), 928-949, 2017. doi: 10.1016/j. ijheh.2017.05.007

Frenoux, C.; Tsitsikalis, A. Domestic private fecal sludge emptying services in Cambodia: between market efficiency and regulation needs for sustainable management. Journal of Water, Sanitation and Hygiene for Development, 5(1), 143-155, 2015. doi: 10.2166/washdev.2014.219

Garrido, J.; Rocha, W.; Gambrill, M.; Collet, H. Estudo de modelos de gestão de serviços de abastecimento de água no meio rural no Brasil: Parte I. Relatório principal. Brasília: Banco Mundial, 2016.

Jordão, E. P.; Pessôa, C. A. Tratamento de esgotos domésticos. Rio de Janeiro: ABES, 2017.
Jung, Y. T.; Narayanan, N. C.; Cheng, Y. L. Cost comparison of centralized and decentralized wastewater management systems using optimization model. Journal of environmental management, 213, 90-97, 2018. doi: 10.1016/j. jenvman.2018.01.081

Langergraber, G.; Pressl, A.; Kretschmer, F.; Weissenbacher, N. Small wastewater treatment plants in Austria-technologies, management and training of operators. Ecological engineering, 120, 164-169, 2018. doi: 10.1016/j.ecoleng.2018.05.030

Larsen, T. A.; Udert, K. M.; Lienert, J. Source separation and decentralization for wastewater management. Recent Advances in Medical Thermology. London: IWA, 2013.

Libralato, G.; Volpi Ghirardini, A.; Avezzù, F. To centralise or to decentralise: An overview of the most recent trends in wastewater treatment management. Journal of Environmental Management, 94(1), 61-68, 2012. doi: 10.1016/j. jenvman.2011.07.010

Lin, H.; Liu, W.; Zhang, X.; Williams, N.; Hu, B. Microbial electrochemical septic tanks (MESTs): An alternative configuration with improved performance and minimal modifications on conventional septic systems. Biochemical Engineering Journal, 120, 146-156, 2017. doi: 10.1016/j. bej.2017.01.003

Machado, A. I.; Beretta, M.; Fragoso, R.; Duarte, E. Overview of the state of the art of constructed wetlands for decentralized wastewater management in Brazil. Journal of environmental management, 187, 560-570, 2017. doi: 10.1016/j.jenvman.2016.11.015

Massoud, M. A.; Tarhini, A.; Nasr, J. A. Decentralized approaches to wastewater treatment and management: Applicability in developing countries. Journal of Environmental Management, 90(1), 652-659, 2009. doi: 10.1016/j. jenvman.2008.07.001

Mesquita, T. C. R.; Santos, T. F. O.; Borges, A. C.; Calijuri, M. L.; Souza, F. M. P.; Rosa, A. P. Decentralized management of sewage using anaerobic systems and its potential to comply with legislation in a developing country: A case study in Brazil. In: 16th World Congress on Anaerobic Digestion. Delft, 23 de jun., 2019.

Morato, S. N. B.; Vieira, Z. C.; Santos, E. S.; Santos, I. L. S. 
Greywater Storage, Treatment and Reuse in Residences. In: International Congress on Engineering and Sustainability in the XXI Century. Cham, 9 de out., 2019.

Oliveira Júnior, J. L. Tratamento descentralizado de águas residuárias domésticas: uma estratégia de inclusão social. In: Lira, W. S.; Cândido, G. A. (Orgs.). Gestão sustentável dos recursos naturais: uma abordagem participativa. Campina Grande: EDUEPB, p. 213-232, 2013.

Opher, T.; Friedler, E. Comparative LCA of decentralized wastewater treatment alternatives for non-potable urban reuse. Journal of Environmental Management, 182, 464-476, 2016. doi: 10.1016/j.jenvman.2016.07.080

Orth, H. Centralised versus decentralised wastewater systems?. Water Science and Technology, 58(5), 259-266, 2007. doi: 10.2166/wst.2007.579

Paffrath, S. F.; Freire, F. B. Comparação entre sistemas centralizado e descentralizado como alternativas para o esgotamento sanitário de Capitão Leônidas Marques/PR. In: XIV Simpósio Ítalo-Brasileiro de engenharia sanitária e ambiental. Porto Alegre, 18 de jun., 2018.

Plansab - Plano Nacional de Saneamento. Decreto $n^{o}$ 8.141, de 20 de novembro de 2013. Dispõe sobre o Plano Nacional de Saneamento Básico - PNSB, institui o Grupo de Trabalho Interinstitucional de Acompanhamento da Implementação do PNSB e dá outras providências. Brasília: DOU de 21/11/2013.

PSBR - Programa Saneamento Brasil Rural. Brasília: Funasa, 2019.

Prasad, C. S.; Ray, I. When the pits fill up: (In)visible flows of waste in urban India. Journal of Water, Sanitation and Hygiene for Development, 9(2), 338-347, 2019. doi: 10.2166/washdev.2019.153

Reymond, P.; Wahaab, R. A.; Moussa, M. S.; Lüthi, C. Scaling up small scale wastewater treatment systems in lowand middle-income countries: An analysis of challenges and ways forward through the case of Egypt. Utilities Policy, 52, 13-21, 2018. doi: 10.1016/j.jup.2018.03.008

Santos, A. B. Caracterização, Tratamento e Gerenciamento de Subprodutos de Correntes de Esgotos Segregadas e Não Segregadas em Empreendimentos Habitacionais. Fortaleza:
Imprece, 2019.

Subtil, E. L.; Sanchez, A. A.; Cavalhero, A. Sistemas descentralizados de tratamento de esgoto e reúso de água. Santo André: UFABC, 2016.

Tonetti, A. L.; Brasil, A.; Madrid, F.; Figueiredo, I.; Schneider, J.; Cruz, L.; Duarte, N. G.; Fernandes, P. M.; Coasaca, R. L.; Garcia, R. S.; Magalhães, T. M. Tratamento de esgotos domésticos em comunidades isoladas: referencial para a escolha de soluções. Campinas: Biblioteca/Unicamp, 2018.

Tchobanoglous, G.; Ruppe, L.; Leverenz, H.; Darby, J. Decentralized wastewater management : challenges and opportunities for the twenty-first century, Water Science and Technology, 4(1), 95-102, 2004. doi: 10.2166/ws.2004.0011

USEPA - United States Environmental Protection Agency. Decentralized Systems Technology Fact Sheet Septage Treatment/Disposal, 1999. Disponível em: < https://www3. epa.gov/npdes/pubs/septage.pdf $>$. Acesso em: jan. 2019.

USEPA - United States Environmental Protection Agency. Handbook for Managing Onsite and Clustered (Decentralized) wastewater treatment systems, 2005. Disponível em: < https://nepis.epa.gov/Exe/ZyPURL.cgi?Dockey=20017K2G.TXT>. Acesso em: jan. 2019.

Von Sperling, M. Introdução à Qualidade das Águas e ao Tratamento de Esgotos: Princípios do Tratamento Biológico de Águas Residuárias. Belo Horizonte: DESA-UFMG, 2014.

WHO - World Health Organization; UNICEF. United Nations Children's Fund. Joint Monitoring Programme. For Water Supply and Sanitation. Data and estimates: tables. Disponível em: <http://www.wssinfo.org/data-estimates/ table/>. Acesso em: nov. 2019.

Yerri, S.; Piratla, K. R. Decentralized water reuse planning: Evaluation of life cycle costs and benefits. Resources, Conservation \& Recycling, 141, 339-346, 2019. doi: 10.1016/j. resconrec.2018.05.016

Zaharia, C. Decentralized wastewater treatment systems: Efficiency and its estimated impact against onsite natural water pollution status. A Romanian case study. Process Safety and Environmental Protection, 108, 74-88, 2017. doi: 10.1016/j.psep.2017.02.004 